with the index attack, and for patients with no further attack the corresponding proportion was $21.6 \%\left(\chi^{2}=1.95 ; 1\right.$ d.f. ; not significant). This result is in keeping with Kellgren's (1938) finding that referred pain can arise from superficial structures in the back, and does not therefore mean that there is pressure on nerve roots. Of the 85 patients with referred pain, only 9 $(10.6 \%)$ had a "proved disc." Moreover, $14(61 \%)$ of the 23 patients with "proved disc" did not experience referred pain.

The duration of the index attack was significant in assessing the liability to recurrence. In the group of patients with no recurrences $68.1 \%$ of index attacks lasted less than two weeks, compared with $55.6 \%$ for the group who did have further attacks $\left(\chi^{2}=5.15 ; 1\right.$ d.f. ; $\left.\mathrm{P}<0.05\right)$.

While the statistical analysis shows that those who had further attacks tended to have longer index attacks, requiring more treatment than was the case with those having no further attack, the tendency was not particularly strong; in other words, this type of information on the index attack is of little value in attempting to identify patients likely to have further attacks. For example, one could use the criterion that any patient whose index attack lasts more than four weeks is liable to a further attack; in this study the duration of the index attack exceeded four weeks for 52 patients, but only $59.6 \%$ of these had a further attack within four years-a figure which is not much larger than the $44.6 \%$ of all patients who had a further attack.

\section{Conclusions and Summary}

Acute lower backache often occurs in general practice. Over a period of four years $7.5 \%$ of the population at risk in the practice studied consulted the doctors with this complaint.
The overall annual inception rate was 24.3 per 1,000 in males and 20.3 per 1,000 in females, but there was considerable variation by age. A peak in both males and females occurred in the 50-59 decade, this age group experiencing twice the overall inception rate.

No cause was evident for the attacks in $79.3 \%$ of males and in $88.8 \%$ of females ; there was a recent history of strain in only $10.9 \%$ of males and $4.3 \%$ of females, and only a small proportion of patients had definite evidence of nerve-root pressure (7.6\% of males and $5.6 \%$ of females).

The majority of the attacks were of short duration, lasting less than two weeks in $62 \%$ of cases. The duration of the attacks was not significantly related to age or sex, nor to the presence of referred pain, but it was related to the presence of objective signs of root pressure. Ninety per cent of the attacks were managed by the general practitioners, who used only symptomatic treatment.

Recurrences were not related to age, sex, or the presence of referred pain, but were more likely to occur in patients diagnosed as having nerve-root pressure and in those whose index attack lasted longer than two weeks.

Of the patients identified by an attack during the incidence study $44.6 \%$ had a recurrence within four years, which is almost four times the rate expected in a group with the same age and sex distribution

REPERENCES

Kellgren, J. H. (1938). Clin. Sci., 3, 175.

General Regíster Office (1954). Medical and Population Subjects Study No. 8. Measurement of Morbidity. H.M.S.O., London.

Stoddard, A. (1959). Manual of Osteopathic Technique. Hutchinson, London.

Troup, J. D. G. (1965). Lancet, 1, 857.
K. T. SHETTY,* M.D. ; N. L. SHARMA, $†$ M.D., D.C.H., D.T.M., D.P.H. ; K. M. WAHAL, $\ddagger$ M.D., D.SC.

Brit. med. F., 1966, 2, 84-86
* Reader in Paediatrics, Kasturba Medical College, Manipal, South India. t Professor of Paediatrics, King George's Medical College, Lucknow, India.

It has been proved that the sex-chromatin body is derived from deoxyribonucleic acid much later than the other and is thus positively heteropyknotic (Ohno et al., 1959). It has also been suggested that one diploid set of autosomes prevents the formation of sex-chromatin mass from one $\mathrm{X}$ chromosome, the Barr body being derived from any additional uninhibited $\mathrm{X}$ chromosome (Ohno et al., 1959 ; Harnden, 1961). On the other hand, Mary Lyon's (1961) hypothesis-that the choice of which of the two X chromosomes is to be inactivated has to be made at a definite point in foetal development, that it is a choice made at random and independently by each cell present at this time, and that once made it is irreversible and transmittable to all descendants of the cell-is very fascinating.

An alteration in the size of the Barr body on antibiotic administration has been reported by Sohval and Casselman (1961). A decreased incidence of sex-chromatin-positive cells in females treated with cortisone has also been reported (Taylor, 1961). We have therefore undertaken a systematic study of the incidence of sex-chromatin mass in cortisone-treated female children.

¥ Reader in Pathology, King Ceorge's Medical College, Lucknow, India. one of the two $\mathrm{X}$ chromosomes in the female which replicates its

\section{Material and Methods}

One hundred healthy girls and 100 healthy boys who had not been on any form of drug therapy for the previous six months were studied as a control group. Sixty-seven female patients of the Children's Hospital, Lucknow, who were being treated with prednisone for various diseases, such as tuberculous meningitis, acute rheumatic carditis, liver-cell failure, hypoplastic anaemia, encephalitis, nephrotic syndrome, enteric fever, bronchopneumonia, etc., were used for the study.

The staining method of Klinger and Ludwig as modified by Barr (1960) was employed. For consistency in results only those nuclei lying against the nuclear membrane were scored as positive.

Two smears were taken from each subject (including the controls) each time the study was made, and 500 cells were counted for each subject and the percentage incidence of sex chromatin was calculated. Whenever too many cells were shrunken, folded, or pyknotic, or if there was much bacterial contamination, fresh smears were examined.

All the slides were coded, and the control slides were mixed with the slides of the prednisone-treated children so that the reading of the slides was "blind." 


\section{Results}

The incidence of sex-chromatin positivity in the male controls varied from 0 to $3 \%$, with a mean frequency of $0.9 \%$; in the female controls it varied from 28 to $64 \%$, with a mean of $42 \%$.

Buccal smears were prepared from each patient before starting therapy and a second set of smears was prepared during therapy, the day on which the second set of smears was prepared differing for different patients without any definite order.

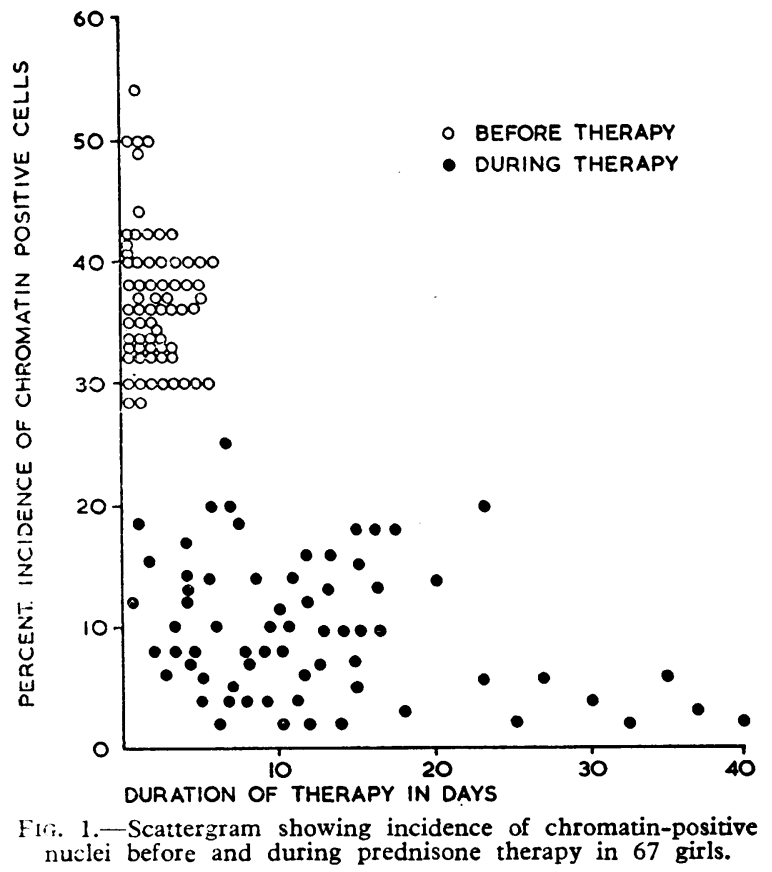

The dosage of prednisone given was $1 \mathrm{mg} . / \mathrm{lb} .(2.2 \mathrm{mg} . / \mathrm{kg}$.) body wcight for all the patients except the four with nephrotic syndrome, who received a slightly higher dosage. Prednisone was used in all except four patients, who received Kenacort (triamcinolone) in an equivalent dosage. In six patients who were on prolonged therapy for more than a month, on a maintenance dose, serial buccal smears were prepared at intervals of four days. In 12 patients buccal smears were also prepared either one or two days after treatment was stopped.

The results show that there is a reduction in the incidence of chromatin-positive nuclei in females on prednisone (Fig. 1), and that this reduction is obvious even one day after prednisone

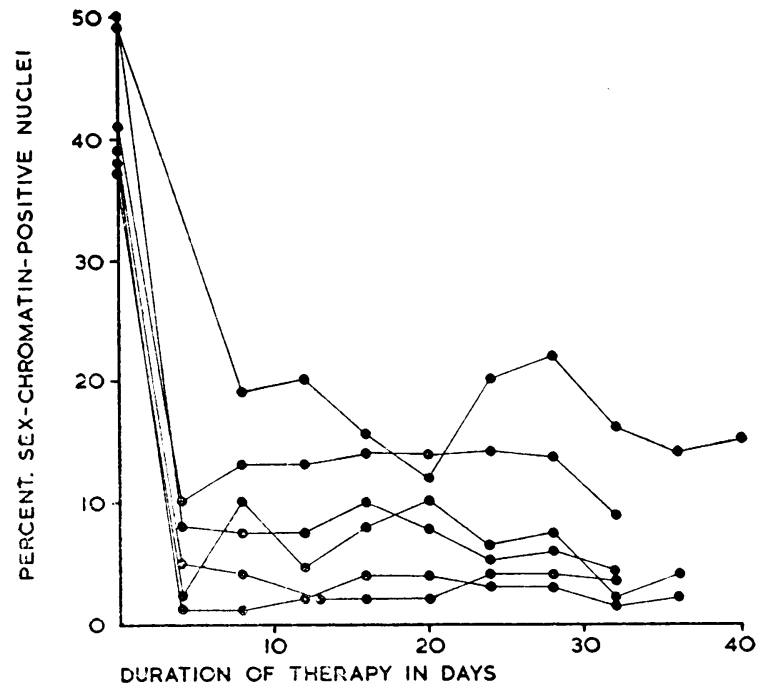

FIG. 2.-Sex-chromatin frequency in six prednisone-treated girls at four-day intervals. is started, while this reduction shows no consistent correlation with the duration of therapy (Fig. 1).

No relation with dosage could be determined, since most of the patients received the same dose, calculated according to body weight. The mean sex-chromatin frequency was $36.9 \%$ before therapy and $9.9 \%$ during therapy. These mean values differ significantly at the $0.1 \%$ level $(\mathrm{P}<0.001)$.

The six patients whose smears were studied at intervals of four days for a period of 32 days show that a rapid initial fall is manifest within the first four days, and this level is maintained approximately at the same level with little fluctuation during the period of treatment (Fig. 2).

The 12 patients in whom buccal smears were prepared either one day or two days after therapy showed a rapid return of chromatin positivity to the normal female range soon after the stopping of therapy (Fig. 3). The mean sex-chromatin frequency was $40.2 \%$ before therapy, $10.1 \%$ during therapy, and $35.3 \%$ after therapy. The mean value during therapy differs significantly from the mean values before therapy and after therapy $(P<0.001)$.

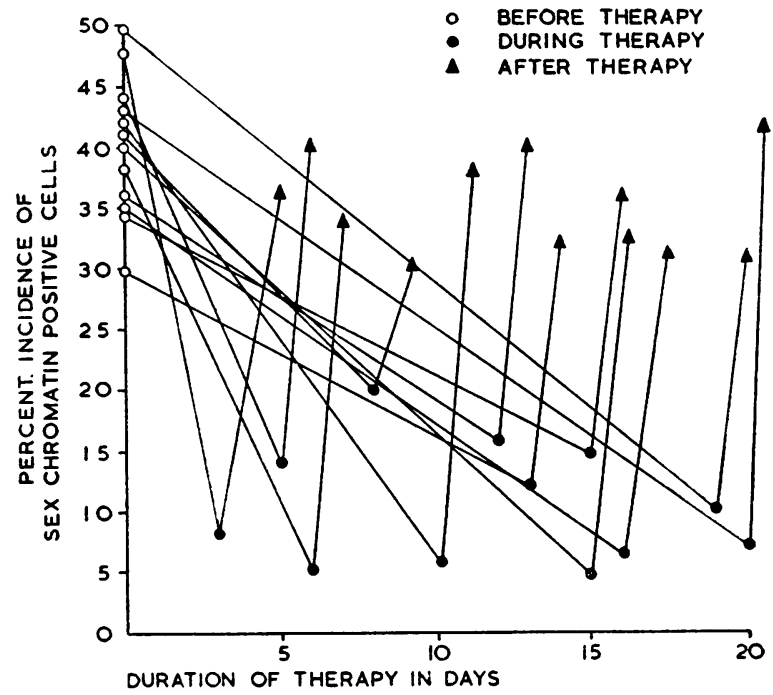

FIG. 3.-Sex-chromatin frequency in 12 prednisone-treated girls before, during, and after therapy.

\section{Discussion}

A reduction in the incidence of the sex-chromatin body which was not uniform and which showed no correlation with the duration of therapy was noted in all the patients. A lowered incidence (at least 50\%) and diffuse chromatin were observed by Taylor (personal communication, 1965) during therapeutic hydrocortisone administration in five cases. Taylor (1963) refers to Jacob's unpublished work, which showed a similar reduction in sex-chromatin frequency during oestrogen therapy.

The results link up well with the decreased incidence found in newborn females (Taylor, 1963), who incidentally have a high oestrogen blood-level (Smith, 1959), and in women during and following menstruation (DelCampo and Ramirez, 1962).

Sohval and Casselman (1961) noted a reduction in the size of the sex-chromatin body when four chemically unrelated antibiotics were given in therapeutic doses, but no change in the prevalence was noted.

From these results it certainly seems that a variety of agents can affect the sex-chromatin body. The results also point out the hazards of misinterpreting chromatin-positive individuals as chromatin-negative during prednisone therapy and the possibility of misinterpreting intermediate counts as indicative of sex-chromosomal mosaicism or partial deletion of the $\mathrm{X}$ chromosome. 


\section{Summary}

In 67 female children receiving therapeutic prednisone nuclear sex determinations were carried out before and during therapy.

In all 67 patients a reduction of the sex-chromatin incidence was noted.

In 12 patients who were studied after therapy was stopped the incidence of sex chromatin was found to return to the normal female range.

In six patients in whom the smears were studied at intervals of four days for 32 days during therapy the sex-chromatin incidence showed a rapid initial fall noticed within the first four days, and then minor fluctuations without further fall during the continuation of therapy.

\section{REFERENCES}

Barr, M. L. (1960). In Recent Advances in Clinical Pathology, edited by S. C. Dyke, Series III, p. 334. Churchill, London.

DelCampo, M. S. B., and Ramirez, O. E. G. (Abstract)-Intersociet Cytology council Inc., Memphis, Tennessee, as cited by Smith, D. W. (1962). Pediatrics, 30,707.

Harnden, D. G. (1961). Lancet, 2,488

Lyon, M. F. (1961). Nature (Lond.), 190, 372.

Ohno, S., Kaplan, W. D., and Kinosita, R. (1959). Exp. Cell. Res., 18 415 .

Smith, $\dot{C}$. A. (1959). In The Physiology of the Newborn Infant, chap. 12. Blackwell, Oxford.

Sohval, A. R., and Casselman, W. G. B. (1961). Lancet, 2, 1386

Taylor, A. I.'(1961). Hum. Chromos. Newsl., 4, 27.

- (1963). Lancet, 1, 912.

\title{
Direct Spinal Anaesthesia for Repair of Myelomeningocele
}

\author{
DENIS G. CALVERT,* M.B., F.R.C.S., F.R.C.S.ED.
}

Brit. med. F., 1966, 2, 86-87

It is now established practice in many of the centres dealing with cases of spina bifida cystica to undertake closure of the lesion within a few hours of birth. This often entails an emergency operation, and a duty anaesthetist who may have little practical experience in dealing with the neonate is presented with a newborn and often premature child to anaesthetize for a major operation in the face-down position.

It is not proposed here to discuss the ethics, economics, and philosophy of operating on these children-I believe, with others (Sharrard, 1963), that operation within the first few hours of life is, at the present time, the correct treatment in cases of myelomeningocele or myelocele.

Operative mortality in these cases is remarkably small, but should be further reduced by: (1) the maintenance of an even body temperature during operation by the use of some form of external warmth to the child (Calvert, 1962); (2) the accurate replacement of blood during operation as it is lost; and (3) avoiding general anaesthesia, which can be technically difficult, and therefore hazardous in inexperienced hands.

Local infiltration anaesthesia is widely used by many surgeons, and has been satisfactorily used by me on numerous occasions, but it has the disadvantages of occasionally producing subcutaneous haematoma formation and inadequate anaesthesia around the flanks owing to the limitation in the amount of local anaesthetic solution that babies will tolerate.

The method of "direct spinal anaesthesia" described below has been used by me during the past year in 26 cases operated on for myelomeningocele situated in the common sites between the lower thoracic region and the sacrum.

\section{Method}

The child, who has not had any form of premedication, is placed prone on some form of warm mattress laid on the operating-table, with a nurse comfortably seated at the head to suckle the child if necessary and to ensure that the airway is kept clear under the towels. A monitoring thermometer lead is placed in the child's rectum and a satisfactory intravenous drip must be running. In this unit an umbilical catheter is

\footnotetext{
- Senior Surgical Registrar, Bristol United Hospitals and South-western
} Regional Hospital Board. inserted in the umbilical vein, and this route permits the rapid administration of blood during operation should this become necessary.

A bacterial culture is taken from the surface of the lesion in order to determine drug sensitivities should evidence of meninigitis present in the post-operative phase. The child's back and the surface of the lesion are prepared with an aqueous chlorhexidine solution. The tincture is used if the medullary plate is covered by an intact membrane.

With a small trocar-pointed blade a puncture wound is made in the peripheral membranes in the midline above the exposed medullary plate. This causes a release of cerebrospinal fluid from the sac and from deep to the medullary plate, so allowing

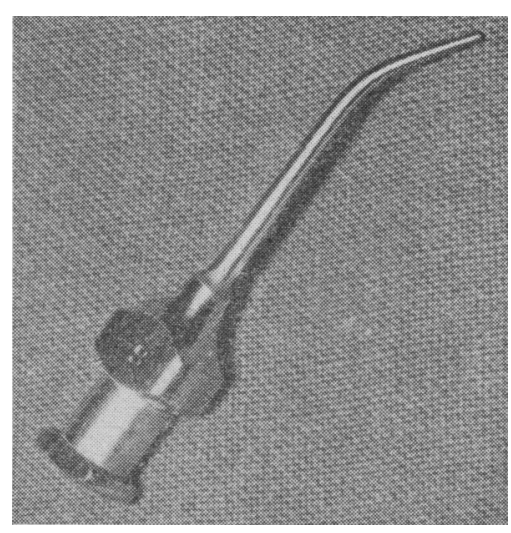

Fig. 1.-Angled Eynards connector.

the latter to lie against the posterior aspect of the vertebral bodies. This small incision usually evokes a transient cry from the child, so demonstrating that the tissues surrounding the lesion are not entirely anaesthetic.

An angled Eynards connector (Fig. 1) attached to a 2-ml. syringe is inserted upwards through the puncture wound, and thus deep to the dura, along the posterior aspect of the spinal cord in the subarachnoid space. The tip of the connector lies about $2 \mathrm{~cm}$. above the upper limit of the exposed medullary plate, and to avoid puncturing the cord the tip is angled 